\title{
Xilooligossacarídeos: produção, aplicações e efeitos na saúde humana
}

\author{
Xylooligosaccharides: production, applications and effects on human health
}

Cristiano Ragagnin de Menezes $^{\mathrm{I}}$ Lucia Regina Durrant $^{\mathrm{II}}$

\section{-REVISÃO BIBLIOGRÁFICA-}

\section{RESUMO}

Os xilooligossacarídeos são açúcares nãoconvencionais (oligômeros formados por unidades de xilose), não-calóricos e não são metabolizados pelo organismo humano. São considerados prebióticos, uma vez que promovem seletivamente o crescimento de probióticos como Lactobacillus sp. e Bifidobacterium bifidum, promovendo uma série de benefícios à saúde humana, como a redução da constipação intestinal, a promoção da digestão e a absorção de nutrientes, a prevenção de infecções gastrintestinais e a inibição do crescimento de microrganismos patogênicos. Este artigo de revisão demonstra a produção de xilooligossacarídeos, tendo como fonte materiais lignocelulósicos, através de métodos químicos e enzimáticos, e também a sua aplicação como ingredientes em alimentos, salientando os efeitos benéficos à saúde provindos desses tipos de compostos.

Palavras-chave: xilooligossacarídeos, prebiótico, efeitos benéficos.

\section{ABSTRACT}

Xylooligosaccharides are non-conventional sugars (sugar oligomers made of xylose units), non-calorics and nondigestible by humans. They are recognyzed as prebiotics once there are non digestible food ingredient that stimulate selectively probiotic growth like Lactobacillus sp. and Bifidobacterium bifidum, promoting several benefices to the human health as the reduction of the gut constipation, the increase of the digestion and nutrient absorption, prevention of gastrointestinal infections, and the inhibition of pathogenic microorganisms. This article reviews the production of xylooligosaccharides from lignocellulosic materials (by chemical or enzymatic methods) and its end products, as well as their application as food ingredients, with special attention to the beneficial effects caused on health by these types of compounds.

Key words: Xylooligosaccharides, prebiotics, health benefits.

\section{INTRODUÇÃO}

Atualmente existe grande preocupação em todo o mundo com a qualidade de vida e a saúde, aumentando assim os cuidados com os alimentos consumidos. Desde então, percebe-se o crescente interesse nos alimentos que apresentam componentes ou substâncias funcionais, ou seja, aqueles que auxiliam ou modulam o bom funcionamento do organismo, de modo a promover a saúde. Estes componentes ou substâncias podem estar presentes naturalmente nos alimentos ou podem ser adicionados aos produtos industrializados.

Em resposta à grande demanda de consumidores por alimentos mais saudáveis e de calorias controladas, grande número de compostos alternativos tem surgido desde o início dos anos 80, entre eles estão os oligossacarídeos. Destes, pode-se destacar os xilooligossacarídeos (XOS), que são importantes devido as suas atividades funcionais.

'Laboratório de Sistemática e Fisiologia Microbiana, Departamento de Ciência de Alimentos (DCA), Faculdade de Engenharia de Alimentos (FEA), Universidade Estadual de Campinas (UNICAMP), CP 6121, 13083-970, Campinas, SP, Brasil. E-mail: ragagnin@fea.unicamp.br. Autor para correspondência.

"Faculdade de Engenharia de Alimentos, UNICAMP, CP 6121, 13083-970, Campinas, SP. 
Em decorrência da ingestão destes compostos, atribuem-se diversos efeitos benéficos à saúde, como, por exemplo, a prevenção de cáries dentárias, a diminuição dos níveis séricos de colesterol total e de lipídeos e o estímulo do crescimento de Bifidobactérias no trato digestivo. Entre os oligossacarídeos utilizados, os XOS possuem a vantagem de serem obtidos através de fontes altamente disponíveis e de baixo custo, como os resíduos florestais e agroindustriais, e de possuírem as propriedades prebióticas semelhantes ou superiores às dos demais compostos, como os frutooligossacarídeos (FOS) e isomaltooligossacarídeos (IOS).

\section{Xilooligossacarídeos: conceitos e produção}

Os xilooligossacarídeos (XOS) são oligômeros de açúcar formados por unidades de xilose, que aparecem naturalmente em frutos, vegetais, leite e mel. Sua produção industrial é obtida através dos materiais lignocelulósicos (LCMs). Os XOS podem ser utilizados para várias finalidades, entre as quais destacam-se as aplicações nos alimentos (VÁZQUEZ et al., 2000 ) e na indústria farmacêutica (VÁZQUEZ et al., 2005). Os materiais lignocelulósicos utilizados para a produção dos XOS são provenientes de uma grande variedade de resíduos, como florestais (madeira de Eucalyptus) e agroindustriais, como sabugo de milho, amêndoas, oliva, cascas de arroz, cevada e aveia (EVTUGUIM et al., 2003; MOURA et al., 2007; NABARLATZ et al., 2007).

Os resíduos agroindustriais estão entre as maiores fontes de biomassa no mundo, representando uma geração anual de 40 milhões de toneladas de resíduo lignocelulósico, gerando considerável prejuízo às atividades econômicas do setor agroindustrial e ao meio ambiente (CANO \& PALET, 2007). Os LCMs são constituídos de três polímeros básicos: lignina (estrutura fenólica), celulose (polímero linear formado por unidades de glicose ligadas na forma $\beta-1-4)$ e a hemicelulose que constituise num heteropolissacarídeo derivado de vários monossacarídeos, incluindo xilose, arabinose, manose e ramnose (GARROTE et al., 1999).

Dependendo da natureza do material lignocelulósico, dos polímeros de xilose (xilana), arabinose (arabinana) ou manose (mananas), podem ser substituídas nas ligações via éter ou éster, formando, por exemplo, o $\alpha$-o-glicopiranosil ácido urônico ou 4-o- metil derivados, grupos acetila e ácidos (EBRINGEROVÁ \& HEINZE 2000; PULS \& SCHUSEIL, 1993; GARROTE et al., 2004). Os materiais típicos para a produção dos XOS são provenientes de uma base rica em xilana, com algumas cadeias heterocíclicas de éter, devendo ser hidrolisada para gerar compostos degradados de cadeia longa (ENEYSKAYA et al., 2007). Estes compostos podem ser produzidos das seguintes maneiras: através de tratamento com enzimas nativas em xilana contida nas LCMs, ou do fracionamento químico de LCM de xilana isolada ou solubilizada, com adição de hidrólise enzimática destes polímeros para XOS, ou pela degradação hidrolítica de xilana para XOS de vapor de água ou por soluções diluídas de ácidos minerais (VÁZQUEZ et al., 2000).

Para a produção direta de XOS a partir de LCM, é preciso que a fração xilana do composto seja separada do complexo lignocelulósico. TAKAO \& YOSHIO (1996) produziram XOS em membranas de polpas de frutas cítricas por métodos enzimáticos (xilana ou fragmentos de xilana solúvel) através de tratamento por álcalis $\left(\mathrm{NaOH}, \mathrm{KOH}, \mathrm{Ca}(\mathrm{OH})_{2}\right.$, amônia ou mistura destes componentes). Este processo é favorecido pela estabilidade do $\mathrm{pH}$ destes polímeros e a fração solubilizada pode ser recuperada através do liquor do processo. Nestes casos, o material cru pode ser preparado com agentes oxidantes, sais e álcoois para remover lignina ou substâncias perdidas. No caso da xilana, pode ser solubilizada em liquor cáustico, precipitado em compostos orgânicos (álcoois, ácidos ou cetonas), seguido de recuperação da hemicelulose dissolvida e de seus produtos de degradação.

Uma vez que a xilana tenha sido isolada ou degradada para a forma solúvel, estará pronta para a hidrólise através de xilanases (VÁSQUEZ et al., 2000; KOSUGI et al., 2002). Para produção de XOS por enzimas, são necessários complexos enzimáticos contendo enzimas exo-xilanases e/ou $\beta$-xilosidases, para evitar a produção de xilose. Estas enzimas podem ser diretamente adicionadas para a reação, imobilizadas ou produzidas in situ por microrganismos (BEG et al., 2001; IEMBO et al., 2001; REZENDE et al., 2002; XIONG et al., 2004; YOON et al., 2006). Através destes métodos, longas cadeias de XOS podem ser produzidas, sendo preferíveis para o uso em alimentos as cadeias entre 24 DP (LOO et al., 1999). Alternativamente, XOS podem ser obtidos através de reações com vapor de água ou água hidróxido catalisada para a degradação de xilana, de acordo com o procedimento conhecido como autohidrólise, hidrotermólise ou água prehidrolisada (CARVALHEIRO et al., 2004; YANG et al., 2005).

Nos primeiros estágios da reação, as espécies catalisadas provêm direto da ionização da água, mas outras reações contribuem para a geração de íons hidrônios em adição de mais estágios da reação. A degradação hidrolítica de hemiceluloses é facilitada quando um ácido é adicionado externamente (prehidrólise ácida), mas, neste caso, os oligossacarídeos 
geram-se em reações intermediárias e a reação principal produz basicamente monossacarídeos (NABARLATZ et al., 2007). Além disso, na degradação da xilana ocorre a solubilização das ligninas ácido-solúveis e a neutralização de cinzas. Todos estes fatores geram quantidades indesejáveis de compostos nãosacarídicos na solução, tornando a etapa seguinte de purificação de extrema importância (VÁZQUEZ et al., 2005).

Para simplificar a purificação dos XOS, a amostra pode ser preparada com solventes antes que ocorra a degradação hidrolítica. Estes solventes de extração promovem de forma mais eficiente a remoção dos extrativos contidos na amostra. Usualmente, estas operações são preferíveis para a extração de altas solubilizações de xilanas, obtendo-se assim XOS com cadeias tipicamente de $\leq 20$. Deste modo, a reação de degradação deve ser evitada, pois no final da reação a distribuição de pesos moleculares não é favorável para a aplicação em alimentos (MOTOHIRO et al., 1986; KABEL et al., 2002).

Xilooligossacarídeos e suas propriedades tecnológicas A utilização de XOS como ingredientes para alimentos funcionais é fundamentada nos seus benefícios para a saúde, incluindo sua estabilidade em longa faixa de $\mathrm{pH}$ e temperatura, metabolismo seletivo para as Bifidobactérias, aumento da produção de ácidos graxos voláteis e redução de lesões de úlcera de estômago (PARAJÓ et al., 2004).

Para aplicação em ingredientes de alimentos, a xilobiose (grau de polimerização=2) é também considerada como um XO\$, sendo que, até mesmo para outros conceitos de "oligo", é associada a compostos de alto grau de polimerização (TATEYAMA et al., 2005). O grau de doçura da xilobiose é equivalente a $30 \%$ em relação à sacarose, sendo que a doçura dos outros XOS é moderada, não possuindo eles efeito residual. Os xilooligossacarídeos são estáveis em pHs entre 2,5 e 8,0, uma vantagem em comparação aos outros oligossacarídeos, como os frutooligossacarídeos (FOS), particularmente na variação de acidez, como os valores baixos de $\mathrm{pH}$ do suco gástrico e temperaturas (acima de $100^{\circ} \mathrm{C}$ ). A atividade de água da xilobiose é reportada como maior do que a de xilose, sendo muito próxima à da glicose. A atividade (antifreezing) de xilobiose na água a temperaturas maiores que $-10^{\circ} \mathrm{C}$ é a mesma da xilose, mas maior que as da glicose, sacarose e maltose (BHAT, 1998).

Como ingrediente em alimentos, os XOS possuem um odor aceitável e são agentes anticarcinogênicos (KAZAMITSU et al., 1997; KAZUYOSHI et al., 1998; HSU et al., 2004), de baixa caloria, sendo utilizados em dietas antiobesidade (TOSHIO et al., 1990; TAEKO et al., 1998). No processamento dos alimentos, os XOS apresentam vantagens sobre a inulina em termos de resistência para os ácidos em aquecimento, sendo utilizados em sucos de baixo pH e bebidas carboidratadas (MODLER, 1994).

Xilooligossacarídeos e seus efeitos para a saúde

Os efeitos dos xilooligossacarídeos para a saúde são principalmente sobre a flora intestinal (JEONG et al., 1998; GIBSON, 2004). Considerável crescimento de Bifidobacterium ssp. no trato gastrintestinal foi obtido in vivo (SUWA et al., 1999), como também no aumento dos ácidos graxos totais de cadeia curta no ceco de ratos (CAMPBELL et al., 1997; IMAIZUMI et al., 1991).

Testes realizados em humanos mostraram que a ingestão de XOS beneficiou a flora intestinal, sendo que a xilobiose $\left(\mathrm{x}_{2}\right)$ não foi excretada nas fezes e urina em 24 horas. Estes compostos não foram hidrolisados pela saliva, pancreatina e pelo suco gástrico, fatores estes que sugerem a utilização dos XOS por bactérias intestinais (OKAZAKI et al., 1991). Estudos realizados com mulheres sofrendo de severos problemas de constipação mostraram que a dieta com XOS reduziu sensivelmente esta constipação, sem apresentar efeitos adversos (TATEYAMA et al., 2005).

A digestibilidade dos XOS no trato gastrintestinal e seus efeitos na absorção de ácidos biliares são comparados aos efeitos dos FOS e dos Isomaltooligossacarídeos (IOS). Considerando-se a digestibilidade, análises de HPLC mostraram a hidrólise de produtos de FOS, IOS, e XOS após quatro horas de digestão in vitro, sendo que a maioria dos IOS e parte dos FOS foram digeridos pelo suco intestinal, porém os XOS não foram digeridos em nenhuma parte por nenhuma enzima digestiva. Os efeitos dos XOS sobre o retardamento da absorção de ácidos biliares comparados com IOS e FOS foram comprovados em experimentos in vitro (JOO et al., 1998). Ensaios "in vitro" provaram que Bifidobacterium spp. e B. adolescentis são hábeis utilizadores de ambos os XOS $\left(\mathrm{x}_{2} \mathrm{e}_{3}\right)$. A absorção de XOS estimula a proliferação de Bifidobacterium bifidum no intestino, porém Staphylococcus, E. coli e espécies de Clostridium spp. não utilizaram os xilooligossacarídeos (SUWA et al., 1999; OKAZAKI et al., 1990).

A maioria das espécies de Lactobacillus utilizam XOS. O L . fermentum demonstrou esta habilidade, mas os Bacterioides utilizaram XOS, em baixa escala, quando comparada à glicose (OKAZAKI et al., 1990). Em comparação com outros 
oligossacarídeos não-digeríveis (NDOs), Bifidobacterium spp. preferiu os XOS, a rafinose e os FOS em relação às hexoses, sendo que os XOS foram mais eficazes do que a rafinose e tão eficazes quanto os FOS em experimentos de crescimento "in vitro" com estas bactérias (JASKARI et al., 1998). Estudos conduzidos por MOURA et al. (2007) demonstraram a eficiência dos XOS, obtidos através da autohidrólise de sabugo de milho, na estimulação do crescimento das culturas probióticas Bifidobacterium adolescentis, B. longum, Lactobacillus brevis e $\boldsymbol{L}$. fermentum, inclusive mostrando ótimos resultados com os XOS de cadeias mais curtas.

Os benefícios comprovados das Bifidobactérias na saúde humana incluem a supressão da atividade de bactérias putrefativas; a prevenção da formação de produtos de nitrosaminas (FUJIKAWA et al., 1991; CAMPBELL et al., 1997); a repressão da proliferação de bactérias patogênicas (SUWA et al., 1999; OKAZAKI et al., 1990; WOLF et al., 1998), devido à produção de ácidos graxos de cadeia curta (ácido acético e lático) (LOO et al., 1999; OKAZAKI et al., 1990; WOLF et al., 1994) e a promoção da digestão e absorção de nutrientes. Isso resulta na habilidade de prevenir infecções gastrintestinais, reduzindo assim a diarréia (DOHNALEK et al., 1998), com aumento do bolo fecal e esvaziamento intestinal periódico nas evacuações (ZIEMER \& GIBSON, 1998; TATEYAMA et al., 2005).

Baseado nos efeitos positivos dos xilooligossacarídeos para a saúde humana, pode-se afirmar que estes compostos são comprovadamente denominados de prebióticos. Por este motivo, são usados como ingredientes ativos em alimentos funcionais (CLYDESDALE, 1997; NABARLATZ et al., 2007; MOURA et al., 2007).

Os XOS são utilizados como ingredientes funcionais no Japão, país cujas suas normas diferem das da América do Norte e Europa. Assim, os japoneses, em vez de denominarem tais compostos de "alimentos funcionais", denominam-nos de FOSHU (Food for Specified Health Use), denominação utilizada desde 1991 (VÁZQUEZ et al., 2000).

\section{CONSIDERAÇÕES FINAIS}

Os xilooligossacarídeos demonstram um grande potencial de utilização em vários produtos, incluindo os farmacêuticos, e em aplicações agrícolas; porém, o maior mercado para o seu desenvolvimento está nos alimentos. Nestes, os XOS apresentam muitas vantagens, se comparados com os outros oligossacarídeos em termos de estabilidade e efeitos à saúde, como o estímulo ao crescimento de probióticos (principalmente Lactobacillus sp. e Bifidobacterium bifidum), juntamente à inibição do crescimento de microrganismos patogênicos, promovendo uma série de benefícios ao organismo.

\section{AGRADECIMENTOS}

À Fundação de Amparo à Pesquisa do Estado de São Paulo (FAPESP), pelo suporte financeiro a este trabalho.

\section{REFERÊNCIAS}

BEG, Q.K. et al. Microbial xylanases and their industrial applications: a review. Applied Microbiology Biotechnology, v.56, p.326-338, 2001.

BHAT, M.K. Oligosaccharides as functional food ingredients and their role in improving the nutritional quality of human food and health. Recent Research Development Agriculture Food Chemistry, v.2, p.787-802, 1998.

CAI, J. et al. Studies on conversion corncobs into xylooligosaccharides by fungi. Weishengwuxue Tongbao, v.24, p.91-94, 1997.

CAMPBELL, J.M. et al. Selected indigestible oligosaccharides affect large bowel mass, cecal and fecal short-chain fatty acids, $\mathrm{pH}$ and microflora in rats. Journal Nutrition, v.127, p.130136, 1997.

CANO, A.; PALET, C. Xylooligosaccharide recovery from agricultural biomass waste treatment with enzymatic polymeric membranes and characterization of products with MALDI-TOFMS. Journal of Membrane Science, v.291, p.96-105, 2007.

CARVALHEIRO, F. et al. Production of oligosaccharides by autohydrolysis of brewery's spent grain. Bioresource Technology, v.91, p.93-100, 2004.

CLYDESDALE, F.M. Aproposal for the establishmentof scientific criteria for health claims for functional foods. Nutrition Research, v.55, p.413-423, 1997.

CRITTENDEN, R.G.; PLAYNE, M.J. Production, properties and applications of food-grade oligosaccharides. Trends Food Science Technology, v.7, p.353-361, 1996.

DOHNALEK, M.I.H. et al. Use of indigestible oligosaccharides to prevent gastrointestinal infections and reduce duration of diarrhea in humans. USA Patent US 5827526, 1998. Capturado em 05 Set. 2006. Disponível na internet: http:// www.freepatentsonline.com/5827526.html.

EBRINGEROVÁ, A.; HEINZE, T. Xylan and xylan derivativesbiopolymers with valuable properties, 1 . Naturally occurring xylans structures, isolation procedures properties. Macromolecular Rapid Communications, v.21, p.542-556, 2000.

ENEYSKAYA, E.V. et al. Biochemical and kinetic analysis of the GH3 family $\beta$-xylosidase from Aspergillus awamori X-100. Archives of Biochemistry and Biophisics, v.457, p.225234, 2007.

EVTUGUIM, D.V. et al. Characterization of an acetylated heteroxylan from Eucalyptus globulus labill. Carbohydrate Research, v.338, p.597-604, 2003. 
FUJIKAWA, S. et al. Efect of xylooligosaccharide on growth of intestinal bacteria and putrefaction products. Journal Japanese Society Nutrition Food Science, v.44, p.37-40, 1991.

GARROTE, G. et al. Antioxidant activity of by products from the hydrolytic processing of selected lignocellulosic materials. Trends in Food Science \& Technology, v.15, p.191-200, 2004.

GARROTE, G. et al. Mild autohydrolysis: an enviromentally friendly technology for xylooligosaccharide production from wood. Journal Chemistry Technology Biotechnology, v.74, p.1101-1109, 1999.

GIBSON, G.R. Prebiotics. Best Practice \& Research, v.18, p.287-298, 2004.

HOWARD, M.D. et al. Dietary fructooligosaccharide, xylooligosaccharide and gum arabic have variable effects on cecal and colonic microbiota and epithelial cell proliferation in mice and rats. Journal Nutrition, v.125, p.2604-2609, 1995.

HSU, C. et al. Xylooligosaccharides and frutooligosaccharides affect the intestinal microbiota and precancerous colonic lesion development in rats. Journal Nutrition, v.134, p.1523-1528, 2004.

KABEL, M.A. et al. Hidrothermally treated xylan rich byproducts yield different classes of xylo-oligosaccharides. Carbohydrate Polymers, v.50, p.47-56, 2002.

KATAHIRA, S. et al. Construction of a xylan-fermenting yeast strain through codisplay of xylanolytic enzymes on the surface of xylose-utilizing Saccharomyces cerevisiae cells. Applied and Environmental Microbiology, v.70, p.54075414,2004

KAZUYOSHI, T. et al. Production of monosaccharide, oligosaccharide and solubilized polysaccharide. Japanese Patent JP 10117800, 1998. Capturado em 13 Set. 2006. On line. Disponível na internet: http://www.freepatentsonline.com /10117800.html.

KAZUMITSU, S. et al. Production of food and drink Japanese Patent JP 9248153, 1997. Capturado em 20 Set. 2006. On line. Disponível na internet: http:// www.freepatentsonline.com /9248153.html.

KOSUGI, A. et al. Xylanase and acetyl xylan esterase activities of xynA, a key subunit of the Clostridium cellulovorans cellulosome for xylan degradation. Applied and Environmental Microbiology, v.68, p.6399-6402, 2002.

IEMBO, T. et al. Production, characterization, and properties of $\beta$-glucosidase and $\beta$-xylosidase from a strain of Aureobasidium sp. Applied Biochemistry and Microbiology, v.38, p.549-552, 2001.

IMAIZUMI, K. et al. Effects of xylooligosaccharides on blood scan. USA Patent US 5939309, 1991. Capturado em 12 Set. 2006. On line. Disponível na internet: http:// www.freepatentsonline.com /5939309.html.

ISAO, Y. et al. Method for obtaining saccharide liquid consisting essentially of xylobiose from hemicellulose liquor. Japanese
Patent JP 1254692, 1989. Capturado em 10 Set. 2006. On line. Disponível na internet: http://www.freepatentsonline.com /1254692.html.

JASKARI, J. et al. Oat $\beta$-glucan and xylan hydrolysates as selective substrates for Bifidobacterium and Lactobacillus Strains. Applied Microbiology Biotechnology, v.49, p.175181, 1998

JEONG, K.J. et al. Hight-level expression of an endoxylanase gene from Bacillus sp. in Bacillus subtilis DB104 for the production of xylobiose from xylan. Applied Microbiology Biotechnology, v.50, p.113-118, 1998

JOO, G.J. et al. Effect of dietary xylooligosaccharide on indigestion and retarding effect of bile acid movement across a dialysis membrane. Han'guk Sikp'um Yongyang Kwahak Hoechi, v.27, p.705-711, 1998.

La GRANGE, D.C. et al. Degradation of xylan to D-xylose by recombinant Saccharomyces cerevisiae coexpressing the Aspergillus niger $\beta$-xylosidase $(x \ln D)$ and the Trichoderma reesei xylanase II (xyn2) genes. Applied and Environmental Microbiology, v.67, p.5512-5519, 2001.

LEMOS, J.L.S. et al. Influence of some sugars on xylanase production by Aspergillus awamori in solid state fermentation. Brazilian Archives of Biology and Technology an International Journal, v.45, p.431-437, 2002.

LOO, J.V. et al. Functional food properties of non-digestible oligosaccharides: a consensus report from the endo project (DGXII-AIRIICT94-1095). British Journal Nutrition, v.81, p.121-132, 1999.

MASAYASU, T. et al. Inhibitor of vasodepressor and vasopressor. Japanese Patent JP 5194241, 1993. Capturado em 5 Set. 2006. On line. Disponível na internet: http:// www.freepatentsonline.com /5194241.html.

MASAYASU, T. et al. Alcoholic fat liver suppresser, Japanese Patent JP 5043470, 1993. Capturado em 25 Set. 2006. On line. Disponível na internet: http://www.freepatentsonline.com /5043470.html.

MODLER, H.W. Bifidogenic factors-sources, metabolism and applications. International Dairy Journal, v.4, p.383-407, 1994.

MOTOHiRo, T. et al. Production of xylose and xylooligosaccharide. Japanese Patent JP 61285999, 1986. Capturado em 12 Set. 2006. On line. Disponível na internet: http://www.freepatentsonline.com /61285999.html.

MOURA, P. et al. In vitro fermentation of xylooligosaccharides from corn cobs autohydrolysis by Bifidobacterium and Lactobacillus strains. LWT - Food Science and Technoology, v.40, p.963-972, 2007.

NABARLATZ, D. et al. Autohydrolysis of agricultural byproducts for the production of xylo-oligosaccharides. Carbohydrate Polymers, v.69, p.20-28, 2007.

OKAZAKI, M. et al. Effect of xylooligosaccharide on growth of intestinal bacteria and putrefaction products. Journal Japonese Society Nutrition Food Science, v.44, p.41-44, 1991. 
OKAZAKI, M. et al. Effect of xylooligosaccharide on the growth of bifidobacteria in bifidobacteria microflora. Journal Japonese Society Nutrition Food Science, v.9, p.77-86, 1990.

PARAJÓ, J.C. et al. Production of xylooligosaccharides by autohydrolysis of lignocellulosic materials. Trends in Food Science \& Technology, v.15, p.115-120, 2004.

PELLERIN, P. et al. Enzymatic production of oligosaccharides from corncob xylan. Enzyme and Microbiology Technology, v.13, p.617-621, 1991.

PULS, J.; SCHUSEIL, J. Chemistry of hemicelluloses: relationship between hemicellulose structure and enzymes required for hydrolysis. Hemicellulose and hemicellulases. (COUGHLAN, M.P.; HAZLEWOOD, G.P., eds.), London, UK: Porland, 1993. p.1-27.

REZENDE, M.I. et al. Xylanase production by Trichoderma rifai by solid state fermentation on sugarcane bagasse. Brazilian Journal of Microbiology, v.33, p.67-72, 2002.

SHUJI, H. et al. Production of xylose and xylooligosaccharide. Japanese Patent JP 62281890, 1987. Capturado em 15 Set. 2006. On line. Disponível na internet: http:// www.freepatentsonline.com /62281890.html.

SUWA, Y. et al. Bifidobacterium bifidum proliferation promoting composition containing xylooligosaccharide. USA Patent US 5939309, 1999. Capturado em 15 Set. 2006. On line. Disponível na internet: http://www.freepatentsonline.com /5939309.html.

TAEKO, I. et al. Food and drink effective in anti-obesity. Japanese Patent JP 10290681, 1998. Capturado em 15 Set. 2006. On line. Disponível na internet: http:// www.freepatentsonline.com /10290681.html.

TAKAO, Y.; YOSHIO, I. Production of gruel-like extract containing xylooligosaccharide and food containing the extract and production of xylooligosaccharide. Japanese Patent JP 8103287, 1996. Capturado em 11 Set. 2006. On line.
Disponível na internet: http://www.freepatentsonline.com / 8103287.html.

TATEYAMA, I. et al. Effect of xylooligosaccharide intake on severe constipation in pregnant women. Journal nutrition Science Vitaminol, v.51, n.6, p.445-448, 2005.

TOSHIO, I. et al. Production of xylobiose. Japanese Patent JP 2119790, 1990. Capturado em 05 Set. 2006. On line. Disponível na internet: http://www.freepatentsonline.com/2119790.html.

VÁZQUEZ, M.J. et al. Xylooligosaccharides: manufacture and applications. Trends in Food Science \& Technology, v.11, p.387-393, 2000.

VÁZQUEZ, M.J. et al. Refining of autohydrolysis liquors for manufacturing xylooligosaccharides: evaluation of operational strategies. Bioresource Technology, v.96, p.889-896, 2005.

XIONG, H. et al. Influence of $\mathrm{pH}$ on the production of xylanases by Trichoderma reesei Rut C-30. Process Biochemistry, v.39, p.729-733, 2004.

YANG, R. et al. Aqueous extraction of corncob xylan and production of xylooligosaccharides. LWT - Food Science and Technoology, v.38, p.677-682, 2005.

YOON, K.Y. et al. Enzymatic production of pentoses from the hemicellulose fraction of corn residues. LWT - Food Science and Technoology, v.39, p.387-391, 2006.

WOLF, B.W. et al. Oral reydratation solution containing indigestible oligosaccharides. USA Patent US 5733579, 1998. Capturado em 15 Set. 2006. On line. Disponível na internet: http://www.freepatentsonline.com /5733579.html.

WOLF, B. et al. Fermentability of selected Oligosaccharides. Federation of American Societies for Experiment Biology, v.8, p.186, 1994.

ZIEMER, C.J.; GIBSON, G.R. An overview of probiotics, prebiotics and synbiotics in the functional food concept: perspectives and future strategies. International Dairy Journal, v.8, p.473-479, 1998. 